\title{
Typhoid Fever Clusters in Kadoma City, Zimbabwe April 2014
}

\author{
Article by Z. Jakopo ${ }^{1}$, D. Chirundu ${ }^{2}$, D. Bangure ${ }^{3,}$ S. Tapesana ${ }^{4}$ \\ ${ }^{1}$ University of Zimbabwe Dept. of Community Medicine, Zimbabwe \\ ${ }^{2}$ Kadoma City Health Dept., Zimbabwe \\ ${ }^{3}$ University of Zimbabwe Dept. of Community Medicine, Zimbabwe \\ ${ }^{4}$ University of Zimbabwe Dept. of Community Medicine, Zimbabwe \\ Email: ${ }^{1}$ zjakopo@gmail.com, ${ }^{2}$ dchirundu@me.com, ${ }^{3}$ bangured@yahoo.com, \\ 4tapesanas@gmail.com
}

\begin{abstract}
Background: Clusters of suspected typhoid cases were recorded in Kadoma City between February 2014 and April 2014. The clusters were at a camp for uniformed forces, a public hospital and a private hospital. Concern by Kadoma residents necessitated the need to investigate the sudden increase in suspected typhoid cases. We then set out to describe the clusters in terms of person, place and time, identify the possible causative organism and source of infection.

Methods: A descriptive cross sectional study was conducted. Interviews with 34 randomly selected cases from the three clusters were done. Municipal water samples were collected for quality analysis. We collected stool specimens and hand swabs from kitchen staff at the 2 hospitals. We also collected kitchen working surface swabs and conducted a sanitary survey.

Results: Males constituted 56\% of the cases. Abdominal pains (32\%), diarrhoea (29\%) and fever (21\%) were common among cases with a positive stool culture. Loss of appetite (26\%), abdominal pains (26\%) and diarrhoea (24\%) were common among cases confirmed by the Widal test. The water treatment plant had quality control measures in place. Two out of 8 hand swabs had E coli isolated and 1 had Salmonella typhi isolated. Four cooks were shedding Salmonella typhi in stool. No faecal coliforms were isolated in the water samples. There were numerous sewer blockages at the uniformed forces camp.

Conclusion: The outbreak was propagated. Food handlers were shedding Salmonella typhi. Hand washing practices were poor. We recommended that the food handlers be removed from the kitchen and monitored. Sewer blockages in the compounds were attended to. Continued surveillance of cases and mobilization of resources in the City was also done.
\end{abstract}

Keywords: Typhoid fever, Clusters, Kadoma City, Zimbabwe

\section{Introduction}

Typhoid fever is a systemic infectious disease caused by Salmonella enterica serotype typhi (Salmonella typhi). The disease is characterized by insidious onset of a sustained nonsweating fever, severe headache, nausea, vomiting, malaise, cough, and rose spots. Constipation is common in adults whilst diarrhoea is common in children (Crump \& Mintz, 2010; Chin, 2000).

Systemic and chronic infections occur and sometimes are not diagnosed. These are asymptomatic carriers and they act as a source of infection. Asymptomatic carriers pose a problem in managing typhoid fever as they spread the bacteria to other individuals (Crump \& Mintz, 2010; Chin, 2000).

Prevention and control of typhoid is centered on avoiding eating contaminated food and proper disposal of excreta. Food handling and preparation has to be monitored. Active case finding is crucial if typhoid is suspected. The source of infection of every case should be ascertained by exploring unreported cases and carriers. Households or close contacts of cases should not be employed as food handlers until there are two negative stool specimens taken 24 hours apart (Ministry of Health and Child Care, 2010; Chin 2000). 
Texila International Journal of Nursing

Volume 3, Issue 1, Mar 2017

An alert threshold for typhoid in Zimbabwe is 1 case and action threshold is 5 cases per 50 000 population. A confirmed typhoid fever case is an individual with laboratory confirmed S.typhi organisms in stool, blood or bone marrow. The drug of choice for treatment of typhoid fever is oral ciprofloxacin (Ministry of Health and Child Care 2010).

Kadoma City has a population of about 96000 people (Zimbabwe National Statistics Agency, 2012). It is a mining and farming town. In Kadoma City, clusters of suspected typhoid cases were recorded in February 2014 at a Uniformed Forces Camp and a Private Hospital. In April food handlers at a Public Hospital were suspected to be suffering from typhoid fever. A meeting held on $22^{\text {nd }}$ April 2014 with stakeholders in Kadoma City highlighted the need to investigate the problem. During the meeting, there were also concerns on the safety of water that is being provided by Kadoma City Council.

It was against this background that we investigated the source of infection in the clusters so as to come up with control measures.

\section{Methodology}

\section{Study design and description of the study site}

We carried out a descriptive cross sectional study. We were given two days to carry out the investigation and a stronger study design could not be conducted. We also wanted to describe the extent of the problem only so as to come up evidence based solutions to the outbreak.

\section{Study subjects and sample size}

The study subjects were people on the suspected typhoid line list. We randomly selected 34 people from the line list for interviews.

\section{Data collection and analysis}

A pretested interviewer administered questionnaire was used to interview 34 randomly selected cases in the three clusters. We collected 13 water samples for water quality analysis at different points along the water distribution system that supplied the three clusters. The water samples were collected using aseptic methods and analyzed for microbiology using the membrane-filtration (MF) method. Turbidity was measured using an electronic Cephalometric turbidity meter and Residual chlorine was measured using a Photo spectrometer. The analysis was done according to the Standard Association of Zimbabwe (SAZ) 560 (1997).

We carried out a sanitary survey of the uniformed forces compound were cases were coming from and the kitchens of the two hospitals. We assessed the water and sanitation facilities available and the state of hygiene of the facilities. We also visited the two municipal treatment plants so as to get an appreciation of the treatment of water and the quality control mechanisms in place.

We collected stool specimens from food handlers at the two hospitals for bacteriological analysis. Specimens were cultured using Selenite F and MacConkey/XLD media. Sub culturing was done onto CLED/XLD media as described in the Zimbabwe Guidelines for Investigation and Management of Typhoid Fever. All samples were transported on the same day to the laboratory and processed within two hours after collection.

We collected hand swabs from food handlers at the two hospitals for bacteriological analysis. Swabs were also collected from the kitchen working tables at the two hospitals. All samples were transported on the same day to a laboratory and processed within two hours after collection. Culturing was done using MacConkey/XLD media.

\section{Ethical considerations}

Written informed consent was sought from the respondents. 


\section{Permission to conduct investigation}

Permission to carry out the study was granted by the authorities responsible for the Uniformed Forces Camp, Private hospital and the Public Hospital.

\section{Results}

We interviewed 34 cases. Males constituted $56 \%$ of the cases. Twenty of the cases tested positive using the Widal test and 14 were confirmed using stool culture.

\section{Signs and symptoms}

Abdominal pains (32\%), diarrhoea (29\%) and fever (21\%) were the most common presenting signs and symptoms among those that had a positive stool culture. Loss of appetite (26\%), abdominal pains (26\%) and diarrhoea (24\%) were the most common signs and symptoms among those that were confirmed using the Widal test. The presenting signs and symptoms of the respondents are shown in Figure 1.

\section{Place}

Among the cases that were interviewed, $50 \%$ of them were coming from the Uniform Forces Camp, 21\% were from the Private Hospital, 18\% were from Rimuka and 12\% from West view areas of Kadoma city.

\section{Time}

In order to get a picture of the nature of transmission, all the 155 cases that were line listed since February 2014 were analyzed to come up with epidemic curves. The cases were confirmed using the Widal test and Stool culture. We constructed two epidemic curves, 1 for the Private Hospital and 1 for the Uniformed Forces Clinic. At the Public Hospital, there were three cases that were confirmed by Widal test out of the 11 food handlers, hence we did not construct an epidemic curve for them.

The first case was recorded on 4 December 2013. Cases started increasing by 5 January 2014 and the outbreak reached three peaks around 21 January 2014, 6 February 2014 and 26 February 2014. The epidemic curve is indicative of a propagated outbreak. The epidemic timeline of the typhoid cases that sought treatment at the private hospital is shown in Figure 2.

The epidemic timeline of the typhoid cases that sought treatment at Uniformed Forces Clinic is shown in Figure 3.The first case was recorded on 20 December 2013. The cases reached a major peak on 22 February 2014. This was the time when the Uniformed Forces Clinic started screening anyone who came to the clinic for typhoid regardless of their illness. The Widal test was used for the screening.

\section{Health seeking behavior}

Median delay in seeking care among the cases was two days with $50 \%$ of the cases seeking care between five and 21 days after onset of illness $\left(\mathrm{Q}_{2}=2, \mathrm{Q}_{1}=5, \mathrm{Q}_{3}=21\right)$. Home treatment was sought by $4(11 \%)$ of the cases, all of which took Oral Rehydration Therapy. At the health facilities, $82 \%$ of the cases were given ciprofloxacin, $41 \%$ paracetamol, $29 \%$ gentamycin and $21 \%$ rocephin (ceftriaxone).

\section{Water, sanitation hygiene}

All the 34 cases had access to municipal water and used a flush toilet. Respondents that resided at Uniformed Forces Camp used communal flush toilets. Soap for hand washing after visiting the toilet was reported to be present at home by $62 \%$ of the cases. In the previous month before their illness $50 \%$ had sewage overflows near their homes. Flies were reported as a problem at households by $47 \%$ of the respondents. 
Texila International Journal of Nursing

Volume 3, Issue 1, Mar 2017

\section{Epidemiological linkages}

Fifty three percent of the cases had contact with someone with a same illness as theirs before they became ill. Only eight cases had travelled outside Kadoma in the month before illness and three had visitors from outside Kadoma before they became ill.

\section{Laboratory results}

The district laboratory did not have the reagents to perform stool culture tests and hence two private laboratories were used. We collected two samples per person /surface and sent them to the two different laboratories for quality control. Among the six hand swabs that we collected two were positive for E coli and one was positive for Salmonella typhi. Two of the eight swabs collected from surfaces were contaminated with $E$ coli. Four out of the 12 stool specimens collected from food handlers were positive for Salmonella typhi. The four food handlers were asymptomatic.

\section{Water quality results}

A total of 13 drinking water samples were collected. Two samples of water were taken soon after water treatment at the treatments works. The other 11 samples were taken at points in the distribution system that supplied the clusters. No faecal coliforms were isolated on bacteriological analysis. Turbidity was within range except at the rigid tanks at the public hospital. Water in these tanks is trucked by Kadoma Municipality every week. The tanks had never been cleaned since their installation. The $p H$ was within range as well as Residual chlorine was slightly below the required minimum of $0.2 \mathrm{mg} / \mathrm{l}$ except at the treatment plant tap where it was $1.64 \mathrm{mg} / 1$. At the treatment plants quality control mechanisms were in place as they also monitored water quality. Along the distribution system water leakages were also noted. The water quality results are shown in Table 1.

\section{Sanitation survey}

We carried out sanitation surveys at Uniformed Forces Camps and the two hospitals.

\section{Uniformed forces camp}

The Uniformed Forces Camp compound had access to municipal water. They use communal toilets that are now old and dilapidated. There were no sanitary disposal facilities in the female toilets. Sewage overflows were noted as shown in Plate 1. There was communal drinking water stand pipes. There was stagnant water at the stand pipes. Solid waste collection was done weekly by the local authority. However, we noted that there were waste heaps at the compound.

\section{Private hospital}

The hospital had access to municipal water and a borehole. There were toilets in the clinic with hand washing facilities. Soap for hand washing was also available.

\section{Public hospital kitchen}

We observed that the kitchen had constant water supply as water was trucked weekly and they also had access to water from the municipal supply. One of the rigid tanks did not have a lid. The kitchen had a toilet facility for staff and a hand washing facility was available. Soap for use after using the toilet was not available.

\section{Discussion}

The three clusters of cases were at the Public Hospital, a Private Hospital and Uniformed Forces Clinic. The cases were confirmed using the Widal test and stool culture, according to the guidelines on managing typhoid fever in Zimbabwe, cases are supposed to be confirmed using a stool culture test. 
Respondents were presenting with abdominal pains and headache which are the signs and symptoms indicative of typhoid fever. Most of the cases were given ciprofloxacin which is the medicine of choice for typhoid cases according to the Zimbabwe guidelines. Sewage overflows and flies were also reported by some of the respondents. Sewage blockages enhance transmission of typhoid as it is transmitted faecal orally. Flies can act as vectors to transmit diseases from faecal matter to food (Chin 2000).

Among the surface and hand swabs we collected there was indication of faecal contamination with salmonella typhi. Poor hand washing practices or use of ineffective disinfectants in the kitchens can lead to contamination of surfaces.

Four of the cooks were shedding salmonella at the time of investigation. This is dangerous as the kitchen would be spreading typhoid fever to the patients and staff that eat food. This is even critical as these kitchens serve food to patients who are already sick. If a cook is found to be shedding Salmonella typhi it is recommended that they be removed from the kitchen and be put on treatment.

Water treatment at the two treatment plants was satisfactory. We were also satisfied with the quality control mechanisms in place at the plants. We also collected water samples for quality analysis. None of the samples that we collected were contaminated with E coli and hence water was ruled out as a possible cause of the problem. The two tanks at the Public Hospital kitchen had high turbidity because they were not being cleaned and had no lids.

Sewage blockages at the uniformed forces compound could perpetuate the outbreaks if they were not attended to on time. Waste heaps could also perpetuate flies' problems in the compound. Flies act as vector for mechanical transmission of typhoid. Maponga et. al. (2013) found out that having garbage near homes $(\mathrm{OR}=1.98$; 95\% CI 1.04-3.77) and flies $(\mathrm{OR}=$ 1.76 ; 95\% CI 1.02-3.02) were risk factors for contracting watery diarrhea in Kadoma.

In the samples analyzed residual chlorine was below the required minimum. Leakages of water pipes can lead to decomposition of residual chlorine such that when water reaches the residents the water would not have the required chlorine residual. Chlorine residual prevents recontamination of water along the distribution system.

\section{Conclusions}

The typhoid fever problem was occurring in three clusters; a Public Hospital, a Private Hospital and Uniformed Forces Camp. Cases were confirmed using the Widal test and stool culture. The place of probable infection of the cases was Kadoma since few travelled outside Kadoma and there were few visitors from outside Kadoma to their households.

Transmission was by person to person transmission. Water treatment at the water treatments plants was satisfactory and there were quality control mechanisms in place. The sanitation survey revealed that there were poor sanitary conditions at the uniformed forces compounds.

\section{Recommendations}

This was a Kadoma City problem and it needed a multidisciplinary approach including both public and private health facilities as well as stakeholders. Typhoid cases should be confirmed using a stool culture test in line with the Zimbabwe guidelines. To the Head of kitchen staff at the two health facilities we recommended immediate removal of the cooks from the kitchen. They had to be put on treatment and monitored until they are cured. To the Engineering Department Kadoma City we recommended attending to sewer blockages and treated water pipe leakages. The communal toilets also needed upgrading with a gender biased focus. We also recommended to the District Environmental Health Officer carrying out health education campaigns at the clusters. To the Director of Health Services Kadoma City we recommended that institutions in the city be monitored and screened including public restaurants. To the District Medical Officer, we recommended capacitating of the District Hospital laboratory. All typhoid cases should be reported to the City Health Department so that they are investigated. 
Texila International Journal of Nursing

Volume 3, Issue 1, Mar 2017

Table1. Water Quality Results Kadoma City, Zimbabwe, April 2014

\begin{tabular}{|c|c|c|c|}
\hline Point of Sampling & $\begin{array}{l}\text { E Coli } \\
\text { (cfu) }\end{array}$ & $\begin{array}{l}\text { Residual } \\
\text { Chlorine (mg/l) }\end{array}$ & $\begin{array}{l}\text { Turbidity } \\
\text { (NTU) }\end{array}$ \\
\hline \multicolumn{4}{|l|}{ Private Hospital } \\
\hline Tap & $0 \mathrm{cfu}$ & 0.17 & 1.97 \\
\hline Borehole & $0 \mathrm{cfu}$ & $\mathrm{n} / \mathrm{a}$ & 0.72 \\
\hline \multicolumn{4}{|l|}{ Uniformed Forces Camp } \\
\hline Tap & $0 \mathrm{cfu}$ & 0.16 & 2.66 \\
\hline Borehole & $0 \mathrm{cfu}$ & $\mathrm{n} / \mathrm{a}$ & 1.13 \\
\hline \multicolumn{4}{|l|}{ Public Hospital } \\
\hline Tank kitchen & $0 \mathrm{cfu}$ & $\mathrm{n} / \mathrm{a}$ & 8.2 \\
\hline Tank Laundry & $0 \mathrm{cfu}$ & $\mathrm{n} / \mathrm{a}$ & 12.9 \\
\hline National Glass Borehole & $0 \mathrm{cfu}$ & $\mathrm{n} / \mathrm{a}$ & 2.07 \\
\hline Private Hospital Tap & $0 \mathrm{cfu}$ & 0.19 & 18.2 \\
\hline Blue Ranges Waterworks & $0 \mathrm{cfu}$ & 0.53 & 1.13 \\
\hline Pasi Waterworks & $0 \mathrm{cfu}$ & 1.64 & 2.78 \\
\hline Waverley Household tap & $0 \mathrm{cfu}$ & 0.15 & 4.54 \\
\hline CBD tap & $0 \mathrm{cfu}$ & 0.13 & 4.63 \\
\hline
\end{tabular}

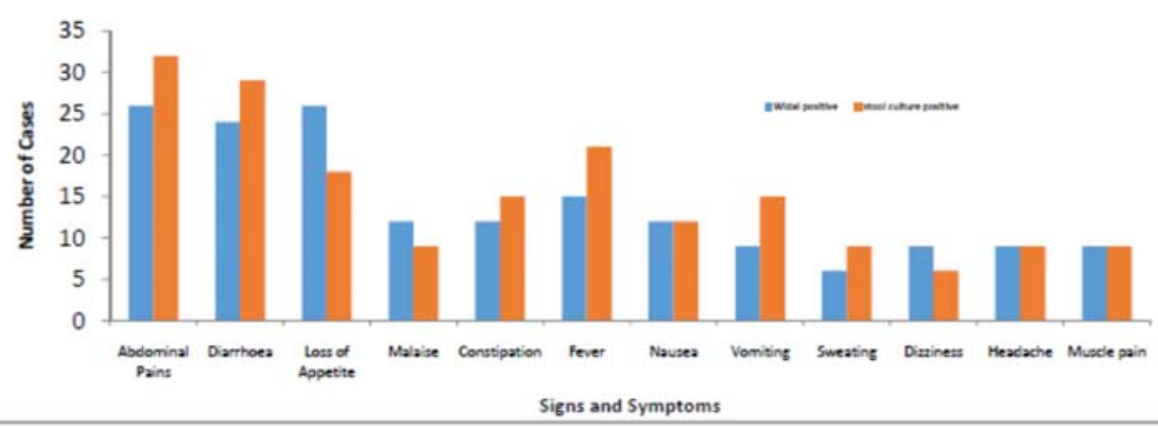

Figure 1. Presenting Signs and Symptoms of the cases by Confirmatory Test Kadoma City, Zimbabwe, 2014

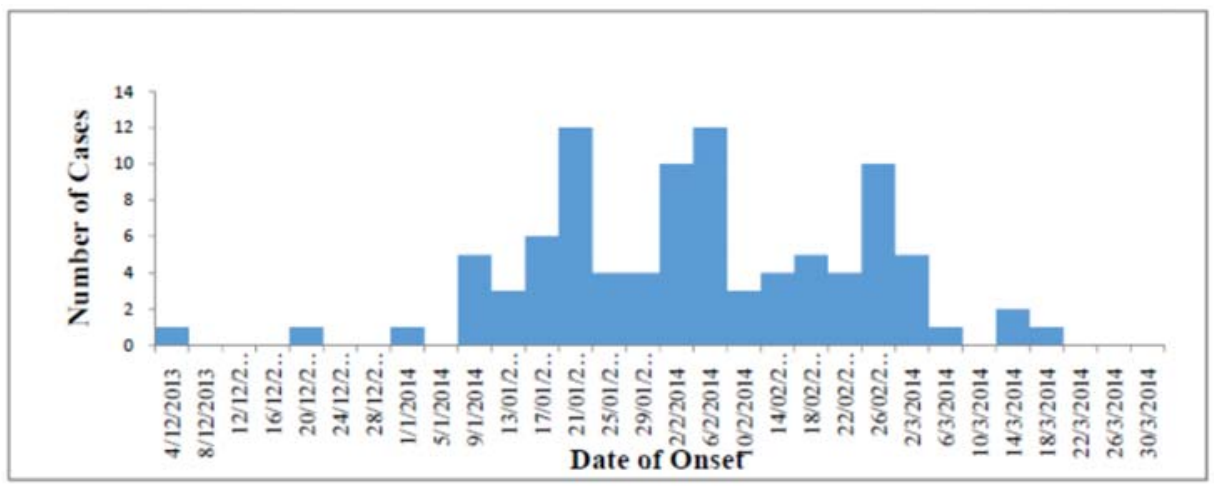

Figure 2. Epidemic Curve Typhoid Cases Private Clinic, Kadoma, City, Zimbabwe, 2014 


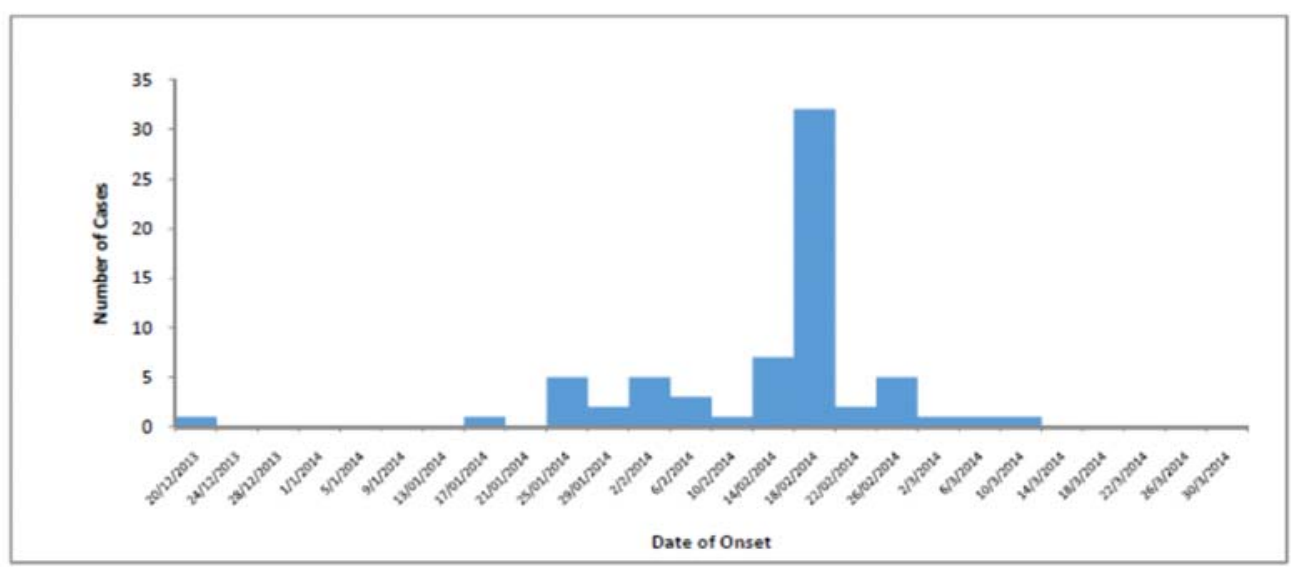

Figure 3. Epidemic Curve Typhoid Cases Uniformed Clinic, Kadoma City, Zimbabwe, 2014

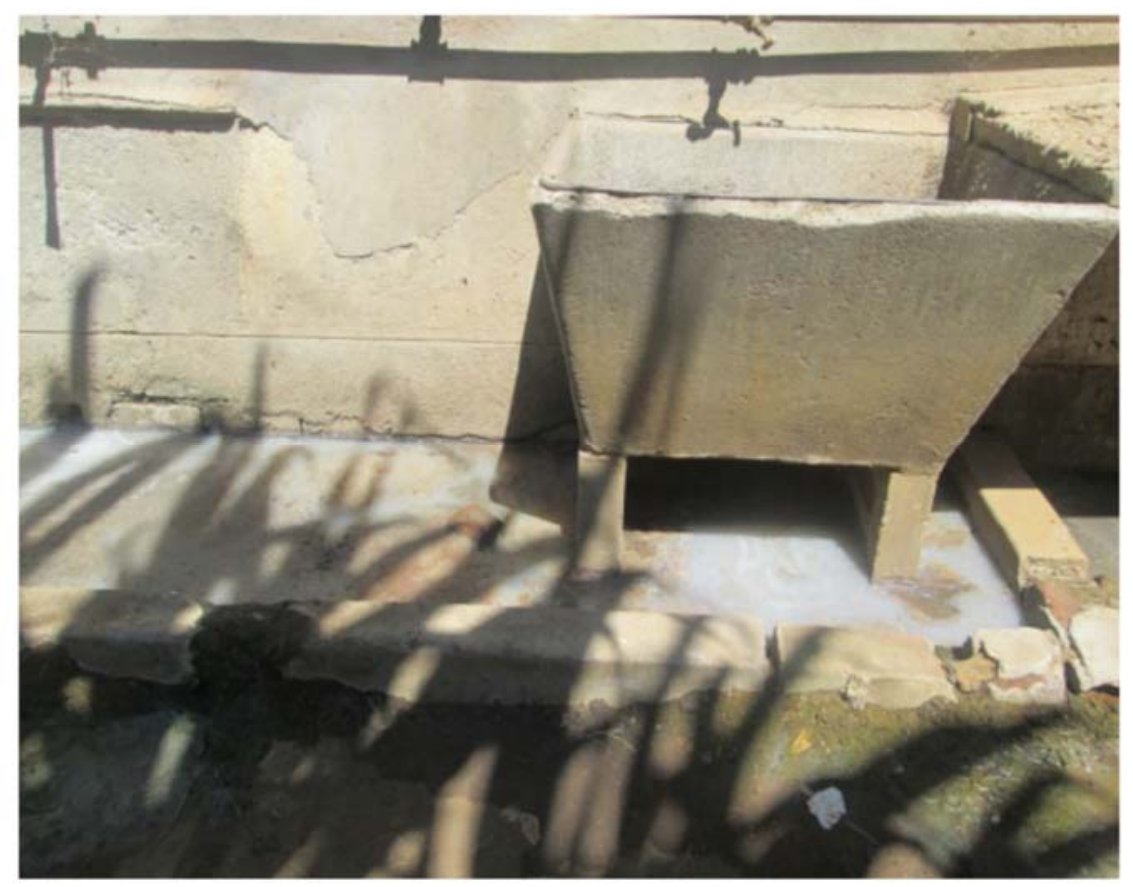

Plate 1. Blocked Sewer behind a communal toilet at the Uniformed Forces Compound

\section{Acknowledgements}

We would like to acknowledge the authorities that helped in availing resources that we used to carry out the investigation. The respondents' participation is also greatly appreciated.

\section{References}

[1]. Crump J A, Luby S P, Mintz E D (2004) The Global burden of typhoid fever Bulletin World Health Organization 82 346-353.

[2]. Crump J.A, Mintz E D (2010) Global trends in typhoid and paratyphoid fever Clinical Infectious Diseases vol 50 (2) pages 241-246.

[3]. Guidelines for the Investigation and Management of typhoid fever in Zimbabwe (2010). Ministry of Health and Child Care Zimbabwe

[4]. James Chin (2000) Control of Communicable diseases Manual17 $7^{\text {th }}$ edition American Public Health Association 
Texila International Journal of Nursing

Volume 3, Issue 1, Mar 2017

[5]. Maponga B, Chirundu D, Gombe NT, Tshimanga M, Shambira G, Takundwa L (2013) Risk factors for contracting watery diarrhea in Kadoma City Zimbabwe 2011 Available at http://www.biomedcentral.com/1471-2334/13/567 Accessed May 2014.

[6]. Standards Association of Zimbabwe (1997) Zimbabwe Standards Specification for Water for Domestic Supplies 560 